\title{
First Significant Digits and the Credit Derivative Market During the Financial Crisis
}

\author{
Paul Hofmarcher ${ }^{1} \&$ Kurt Hornik ${ }^{2}$
}

ABSTRACT

\begin{abstract}
The Credit Default Swap (CDS) market has both been lauded for its ability to stabilize the financial system through credit risk transfers and been the source of regulatory concern due to its size and lack of transparency. As a decentralized over-the-counter market, detailed information about pricing mechanisms is rather scarce. To investigate reported CDS prices (spreads) more closely, we make use of empirical First Significant Digit (FSD) distributions and analyze daily CDS prices for European and US entities during the financial crisis starting in 2007. We find that on a timeaggregated level, the European and US markets obey empirical FSD distributions similar to the theoretical ones. Surprising differences are observed in the development of the FSD distributions between the US and European markets. Whereas the FSD distribution of the US derivative market behaves nearly constantly during the last financial crisis, we find huge fluctuations in the FSD distribution of the European market. One reason for these differences might be the possibility of strategic default for US companies due to Chapter 11 and avoided contagion effects.
\end{abstract}

KEY WORDS: $\quad$ Benford's law; first significant digits; CDS market; financial crisis

JEL Classification: G01

${ }^{1}$ Johannes Kepler University Linz, Austria; ${ }^{2}$ Vienna University of Economics and Business, Austria

\section{Introduction}

The financial markets and the world economy as a whole are currently beset by huge uncertainty. What was sparked by a decrease of housing prices in the US eventually led to a near collapse of the global credit markets. In this article, we use empirical first significant digits (FSD) and theoretical Benford-like distributions (Benford, 1938; Grendar, Judge, \& Schechter, 2007) to study the credit default swap (CDS) market during the financial crisis, which started in July 2007. The reason we choose the CDS market as representa-

Correspondence concerning this article should be addressed to: Paul Hofmarcher, Department of Applied Statistics, Johannes Kepler University Linz, Altenbergerstraße 69 , Linz 4040, Austria, e-mail: paul.hofmarcher@jku.at tive of the credit market is twofold: First, before the crisis, the CDS market was often lauded as an overthe-counter (OTC) market with prodigious risktransferring ability, which stabilizes the financial system as a whole (Greenspan, 2005). Second, from being a fledgling market in the mid-nineties, the CDS market has grown tremendously over the last decade (Dechert LLP, 2008) and is currently an integral part of the financial system.

This work has two main findings: First, we illustrate the usefulness of FSD distributions to verify the "quality" of such data "in some vague sense" (George \& Laura, 2009; Varian, Morgan, Deaton, Cramer, \& Bibby, 1972). Particularly for the CDS market, this finding is essential because the CDS market is a decentralized OTC market and is often pictured as an opaque market with little information about pricing mechanisms, 
price setters and traded volumes. We find that for $\mathrm{Eu}-$ rope and the US, the first digits follow an FSD distribution pattern similar to the proposed Benford-like FSD distribution; that is, the appearance of the first digits follow a weakly monotonic decreasing pattern as provided by (Benford, 1938; Grendar et al., 2007).

Secondly, provided with daily data, we are interested in the development of the FSD distributions during the financial crisis of 2007. Here we find huge differences between the FSD distribution of US companies and that of European ones. Quite surprisingly, for US companies, the FSD distribution of the CDS spreads remained nearly stable during the financial crisis, which poses the question of why the CDS market of this region - which was the origin of the financial crisis - is more stable than the European market in terms of its FSD distribution.

This article is organized as follows: Section II briefly reviews the literature on FSD distributions and the CDS market. Section III describes our data. Section IV presents the main results of this article. Finally, our conclusions and discussions about further research follow.

\section{Benford's Law, Benford-like Distributions and the CDS Market}

Benford's Law (Benford, 1938) is an unexpected mathematical relationship that states that the FSDs of numerous examples of data follow a specific distribution and are not uniformly distributed, as one would expect. It postulates that the probability that the first digit is $\mathrm{i}=\{1 \ldots 9\}$ is given by $\mathrm{p}(\mathrm{i})=\log 10(1+1 / \mathrm{i})$.

For example, 1 appears approximately $30.1 \%$ of the time as the first digit, and 9 appears only $4.6 \%$ of the time. Nearly 60 years later, (Hill, 1995) provides a rigorous proof of this law as well as the conditions under which it holds. Today, a wide range of data sets have been tested according to Benford's Law, e.g., (Clippe \& Ausloos, 2012; Depken, 2008; Giles, 2007; Günnel \& Todter, 2009) or (Ley, 1996). Ley (1996), for example, finds that 1-day returns of the S\&P 500 are reasonably consistent with Benford's Law. In a similar vein, Corazza, Ellero, \& Zorzi, (2010) discuss the S\&P 500 stock market. Inter alia, they find that sequences of trading days not fulfilling the Benford distribution are rather short. Huge deviations from Benford's Law are often interpreted as signals of some type of irregularity, like psychological price barriers or price collusions (DeCeuster, Dhaene, \& Schatteman, 1998; Giles, 2007). However, the literature also shares the common result that we rarely find a perfect match of the observed FSDs to Benford's Law. As noted by Scott and Fasli (2001), only approximately one half of Benford's original data sets provide reasonably close fits to the Benford distribution. Therefore, Grendar et al. (2007) propose an information theoretic approach based on the first moments of the empirical FSDs to derive modifications of the Benford distribution - Benford-like distributions. The idea of this approach is to estimate a probability distribution $\mathrm{R}$ that minimizes the Kullback-Leibler distance to the Benford distribution and has a first moment equal to the empirical FSD mean. Distributions for different first moments of FSDs are tabulated in Grendar et al. (2007). The resulting Benford-like distribution R provides a null distribution for testing empirical FSD distributions.

A broad range of literature analyzes the CDS market, e.g., Forte and Peña (2009); Longstaff, Mithal, and Neis (2005); Realdon (2008). Jorion and Zhang (2007) study contagion effects in the CDS market due to Chapter 11 and Chapter 7 events.

Building on the work of Ley (1996), who studied the stock market using Benford's Law, and Realdon (2008), who linked the CDS market to the stock market, we study the CDS market using FSD and Benford-like distributions.

\section{Data}

As a basis to illustrate the distribution of the FSDs and to check the appropriateness of FSD distributions for studying the OTC market, we use daily Markit CDS data for European and US companies. Markit is one of the leading data providers specializing on pricing credit derivatives. According to Markit, the CDS spreads do not represent the actual traded spreads. Instead, each contributor to Markit provides data from its books of records and/or automated trading systems. The offered Markit CDS spreads are composites of these different sources. In this work, we use daily CDS spreads in basis points (bpts.) for European and US companies that run from 2006-08 to 2010-02. We are provided with spreads for 11 different maturities ranging from 6 months to 30 years.

In addition to basic CDS contract terms, such as maturity, sector and issuer, CDS contracts of- 
Table 1. Descriptive Statistics of CDS spreads in the sample

\begin{tabular}{lcccc}
\hline & \multicolumn{2}{c}{ Investment } & \multicolumn{2}{c}{ Subinvestment } \\
\cline { 2 - 4 } & Europe & US & Europe & US \\
\hline Mean CDS spread (basis points) & 79.778 & 83.095 & 166.647 & 227.563 \\
Median CDS spread & 66.976 & 57.456 & 118.701 & 146.479 \\
Standard Deviation of CDS spreads & 49.598 & 73.086 & 144.491 & 226.026 \\
\hline
\end{tabular}

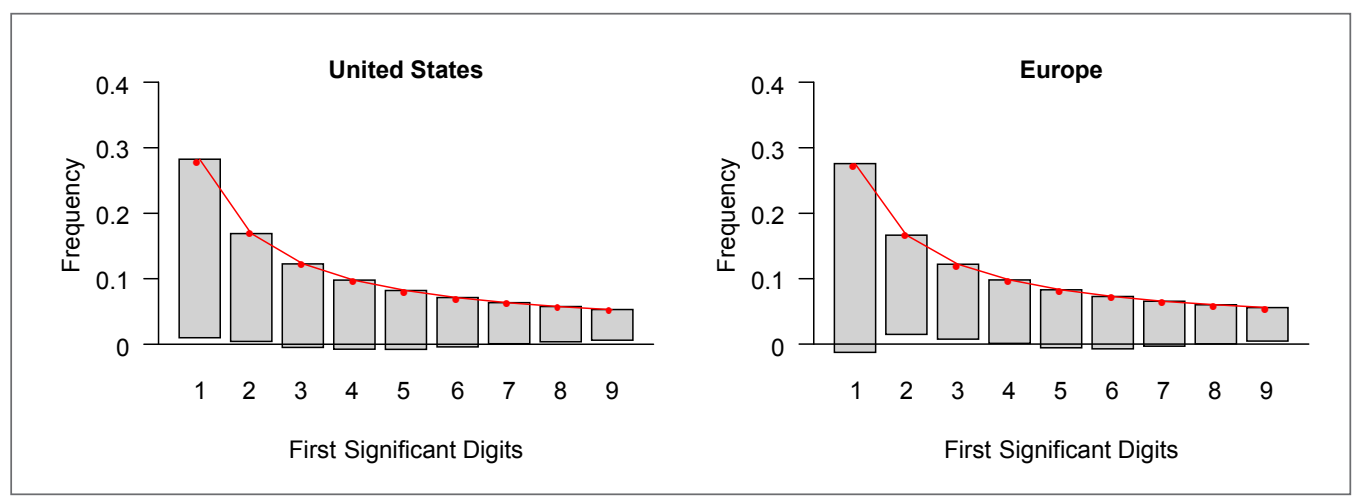

Figure 1. Time-aggregated rootograms for the US and European CDS market. The FSD means $\mathrm{d}^{-}$are 3.6 for the US and 3.66 for Europe.

ten come in four different flavors according to their restructuring mechanism (no restructuring (XR), full restructuring (CR), modified restructuring (MR), or modified-modified restructuring (MM)). The Markit data set contains CDS spreads in all four different restructuring versions. For studying FSD distributions, we do not exclude any of these restructuring mechanisms.

Our time series runs 868 days, which results in a total of $1.44 \times 108$ CDS spread observations.

For the different regions, we observe, on a daily average, 78, 289 observations for European companies and 88,346 for US ones. Table 1 summarizes some descriptive statistics of the CDS spreads for the European and the US market. It contains the observed means, medians and standard deviations for the considered time series. We split the data into two categories, investment CDS entities and subinvestment CDS entities. The first group contains entities with a credit rating - the av- erage of the Moody's and S\&P ratings, provided by Markit - of at least A, and the second group contains all CDS entities rated worse than $\mathrm{A}$.

\section{Results}

Reasonability of the data: To verify the quality of our data, we use the approach proposed by Grendar et al. (2007). Fig. 1 presents rootograms (Tukey, 1972) for the aggregated daily FSD distributions of US (left) and European (right) CDS entities. A rootogram is a modified version of a histogram. The observed proportions are displayed as bars "hanging" on the estimated Benfordlike distributions $\mathrm{R}$ that are calculated on the basis of the observed FSD means for the different regions. Hanging the bars of the observed frequencies on the estimated reference distribution $\mathrm{R}$ has the advantage of allowing us to judge the differences more easily against a horizontal line. As we can see for both markets, the FSD distributions are very similar to the theoretical ones. 
Table 2. Kuiper test results.

\begin{tabular}{ccc}
\hline & US & Europe \\
\hline sample size N & 76684486 & 67954791 \\
Kuiper V & 0.02462 & 0.02382 \\
\hline
\end{tabular}

For both markets, statistical tests like a $\chi^{2}$-test or Kuiper test would reject the null-hypothesis because of the huge power that any of these tests have given the large sample size. If one takes models as approximations to reality, instead of perfect data reproducers, this can be seen as a weakness of Neyman Pearson statistics (Ley, 1996). For sample sizes $\mathrm{N}$ less than 5000 observations, we would not reject the null at a significance level of $1 \%$. Table 2 summarizes the Kuiper's basic test statistics V (Giles, 2007). Kuiper's test is closely related to the Kolmogorov Smirnov test and compares the cumulative distribution functions of interest with the cumulative distribution of the reference distribution. The test statistic is given as the sum of the maximum distance above (D+) and below (D-) the cumulative distribution functions that are compared, i.e., $\mathrm{V}=\mathrm{D}+$ + D- (Kuiper, 1960).

Empirical FSD development through the crisis: Because we are interested in the development of the empirical FSD distributions during the financial crisis, Fig. 2 displays the FSD of US (top) and European (bottom) CDS spreads. The subplot at the left margin of each plot illustrates the theoretical values of the reference distributions $\mathrm{P}$. The vertical lines in the graphs indicate key events in the 2007 financial crisis. The first vertical line denotes the start of the crisis - July 17, 2007 - when Bear Stearns disclosed that two of its subprime hedge funds had lost nearly all of their value. The second line - March 16, 2008 - indicates the Fire Sale of Bear Stearns to JP Morgan, and the last line indicates the collapse of Lehman Brothers (September 15, 2008). Fig. 2 shows that the FSD distribution for the US CDS market remains nearly constant. The lines in the graphs denote the empirical proportions of the single digits. From top to bottom, the first line stands for digit 1 ; that is, at the beginning of our time series, 1 appeared as the first digit with 0.247 for European CDS spreads and with 0.257 for US CDS spreads. For digit
2, we observe 0.177 and 0.175 at the first observation date for the European and US markets, respectively. For digit 9, 0.037 and 0.042 are observed. Taking the default of two Bear Stearns Hedge Funds as the beginning of the subprime crisis, we see from Fig. 2 that before the crisis, the American and European market behave very similarly. However, following the beginning of the crisis, both markets develop completely differently in terms of FSD distributions. Whereas the US market remains more or less stable, with a standard deviation for the 1 as a leading digit during the whole time period of only 0.02, we observe for the European market and digit 1 , a standard deviation more than twice as large, namely 0.05 .

A well known difference between the US and European CDS market is their composition in terms of credit quality. The US CDS market is broader in the sense of credit quality; that is, we observe more bad credit quality CDS entities. To take this fact into account, we split our data into "investments" and "subinvestments". The first group only contains entities with an average credit rating quality of at least A, whereas the second group contains all non-default entities below A, i.e., from BBB to CCC/C. The results for these two groups are illustrated in Fig. 3. As we can see in both groups - investment and subinvestment companies - the FSD distribution for US entities is more stable. The higher FSD fluctuation of the investment-grade CDS market is caused by the fact that spreads are more concentrated at the lower end of the spread scale. That is, we mainly observe spreads below 100 bpts. Therefore, a change of the credit spreads is more likely to come along with a change in the FSD than for spreads above, e.g., 100bpts. However, as we can see from Figure 3, splitting the data into investment and subinvestment grades does not sufficiently explain the regional differences in the FSD distributions. 


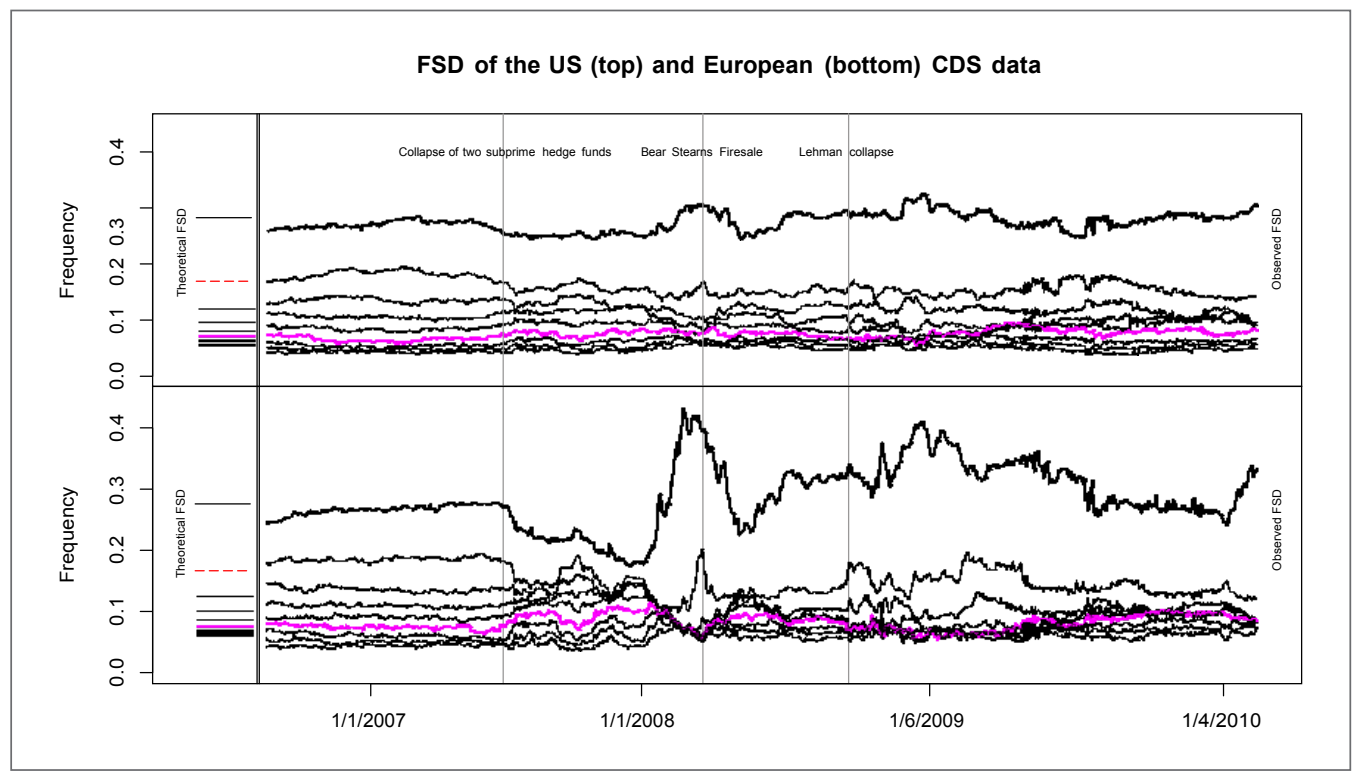

Figure 2. FSD of the CDS spreads of US and European Companies. From the top down, the lines indicate the frequencies of the leading digits in increasing order, i.e., from 1 to 9.

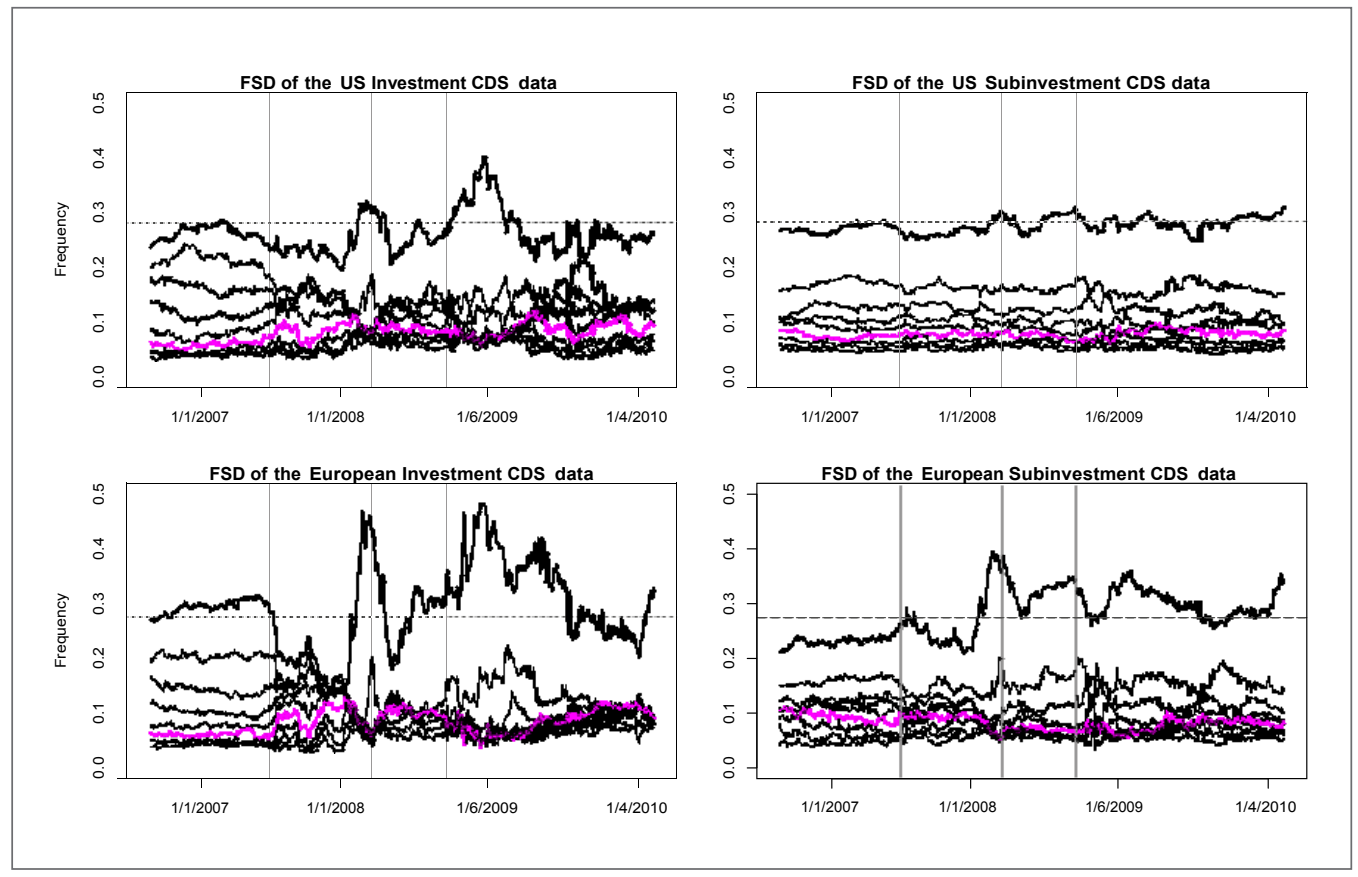

Figure 3. FSD of the investment and subinvestment grade CDS spreads. The vertical lines indicate key events of the financial crisis. The dashed horizontal lines denote the theoretical levels of 1 as a leading digit. 
Empirical FSD for different restructuring mechanism of the CDS contracts: In search for potential explanations for the regional differences mentioned above, we investigate the FSD distributions of the CDS spreads according to their restructuring clauses, which are a key feature of every CDS contract, defining the credit events that trigger default. Table 3 presents summary statistics for the different document clauses for both European and US CDS spreads. We can infer from Table 3 that the proportion of traded CDS contracts with either full restructuring (CR) or modifiedmodified restructuring (MM) is much higher for European CDS entities than for US ones. More than two thirds of all traded European CDS entities belong to either CR or MM. For the US market, we find a majority - nearly two thirds - of traded CDS entities belonging to the other document clauses, modified restructuring (MR) and no restructuring (XR).

Table 3. Proportions of restructuring mechanisms in the CDS data.

\begin{tabular}{lcccc}
\hline Europe & & & & \\
\hline Restructuring Clause & CR & MM & MR & XR \\
Proportion & 0.3216936 & 0.4375637 & 0.1596982 & 0.0810445 \\
\hline US & & & & \\
\hline Restructuring Clause & CR & MM & MR & XR \\
Proportion & 0.1892917 & 0.1468002 & 0.4250354 & 0.2388727 \\
\hline
\end{tabular}

Table 4. FSD distributions for European CDS according to their restructuring mechanisms.

\begin{tabular}{|c|c|c|c|c|c|c|c|c|c|}
\hline Digits & 1 & 2 & 3 & 4 & 5 & 6 & 7 & 8 & 9 \\
\hline \multicolumn{10}{|c|}{ MR - Modified Restructuring } \\
\hline pre 2007/07/17 & 0.27 & 0.18 & 0.13 & 0.10 & 0.09 & 0.07 & 0.06 & 0.05 & 0.05 \\
\hline 2007/07/17-2008/03/16 & 0.25 & 0.14 & 0.12 & 0.11 & 0.10 & 0.09 & 0.07 & 0.06 & 0.05 \\
\hline 2008/03/16-2008/09/15 & 0.31 & 0.13 & 0.10 & 0.09 & 0.09 & 0.09 & 0.07 & 0.07 & 0.06 \\
\hline 2008/09/15 - & 0.32 & 0.15 & 0.10 & 0.08 & 0.08 & 0.08 & 0.07 & 0.06 & 0.06 \\
\hline \multicolumn{10}{|l|}{ XR - No Restructuring } \\
\hline pre 2007/07/17 & 0.27 & 0.18 & 0.14 & 0.11 & 0.09 & 0.07 & 0.06 & 0.04 & 0.04 \\
\hline 2007/07/17-2008/03/16 & 0.24 & 0.14 & 0.12 & 0.11 & 0.10 & 0.09 & 0.07 & 0.06 & 0.05 \\
\hline 2008/03/16-2008/09/15 & 0.30 & 0.13 & 0.10 & 0.10 & 0.09 & 0.09 & 0.07 & 0.07 & 0.06 \\
\hline 2008/09/15 - & 0.32 & 0.14 & 0.10 & 0.08 & 0.08 & 0.08 & 0.07 & 0.07 & 0.06 \\
\hline \multicolumn{10}{|c|}{ CR - Complete Restructuring } \\
\hline pre 2007/07/17 & 0.27 & 0.18 & 0.14 & 0.11 & 0.09 & 0.07 & 0.06 & 0.05 & 0.04 \\
\hline 2007/07/17-2008/03/16 & 0.24 & 0.15 & 0.12 & 0.12 & 0.10 & 0.09 & 0.07 & 0.06 & 0.05 \\
\hline 2008/03/16-2008/09/15 & 0.30 & 0.13 & 0.10 & 0.09 & 0.09 & 0.08 & 0.07 & 0.06 & 0.05 \\
\hline 2008/09/15 - & 0.32 & 0.14 & 0.10 & 0.08 & 0.08 & 0.08 & 0.07 & 0.07 & 0.06 \\
\hline \multicolumn{10}{|c|}{ MM - Modified-Modified Restructuring: } \\
\hline pre 2007/07/17 & 0.27 & 0.18 & 0.14 & 0.11 & 0.09 & 0.07 & 0.06 & 0.05 & 0.04 \\
\hline 2007/07/17-2008/03/16 & 0.24 & 0.15 & 0.12 & 0.12 & 0.11 & 0.09 & 0.07 & 0.06 & 0.04 \\
\hline 2008/03/16-2008/09/15 & 0.29 & 0.13 & 0.10 & 0.10 & 0.10 & 0.08 & 0.07 & 0.07 & 0.05 \\
\hline 2008/09/15 - & 0.31 & 0.14 & 0.10 & 0.09 & 0.08 & 0.08 & 0.08 & 0.07 & 0.06 \\
\hline
\end{tabular}


Table 5. FSD distributions for US CDS spreads according to their restructuring mechanisms.

\begin{tabular}{|c|c|c|c|c|c|c|c|c|c|}
\hline Digits & 1 & 2 & 3 & 4 & 5 & 6 & 7 & 8 & 9 \\
\hline \multicolumn{10}{|c|}{ MR - Modified Restructuring } \\
\hline pre 2007/07/17 & 0.27 & 0.18 & 0.14 & 0.11 & 0.09 & 0.07 & 0.06 & 0.05 & 0.04 \\
\hline 2007/07/17-2008/03/16 & 0.26 & 0.16 & 0.13 & 0.11 & 0.10 & 0.08 & 0.07 & 0.06 & 0.05 \\
\hline 2008/03/16-2008/09/15 & 0.28 & 0.15 & 0.12 & 0.10 & 0.09 & 0.08 & 0.07 & 0.06 & 0.05 \\
\hline 2008/09/15 - & 0.29 & 0.16 & 0.12 & 0.10 & 0.09 & 0.08 & 0.07 & 0.06 & 0.05 \\
\hline \multicolumn{10}{|l|}{ XR - No Restructuring } \\
\hline pre 2007/07/17 & 0.28 & 0.20 & 0.14 & 0.10 & 0.08 & 0.06 & 0.05 & 0.04 & 0.04 \\
\hline 2007/07/17-2008/03/16 & 0.25 & 0.17 & 0.14 & 0.12 & 0.09 & 0.08 & 0.06 & 0.05 & 0.04 \\
\hline 2008/03/16-2008/09/15 & 0.26 & 0.16 & 0.13 & 0.11 & 0.09 & 0.07 & 0.06 & 0.06 & 0.05 \\
\hline 2008/09/15 - & 0.28 & 0.16 & 0.12 & 0.10 & 0.09 & 0.08 & 0.07 & 0.06 & 0.05 \\
\hline \multicolumn{10}{|c|}{ CR - Complete Restructuring } \\
\hline pre 2007/07/17 & 0.27 & 0.18 & 0.14 & 0.11 & 0.09 & 0.07 & 0.05 & 0.05 & 0.04 \\
\hline 2007/07/17-2008/03/16 & 0.26 & 0.16 & 0.13 & 0.11 & 0.10 & 0.08 & 0.07 & 0.06 & 0.05 \\
\hline 2008/03/16-2008/09/15 & 0.29 & 0.15 & 0.12 & 0.10 & 0.09 & 0.08 & 0.07 & 0.06 & 0.05 \\
\hline 2008/09/15 - & 0.28 & 0.16 & 0.12 & 0.10 & 0.09 & 0.08 & 0.07 & 0.06 & 0.05 \\
\hline \multicolumn{10}{|c|}{ MM - Modified-Modified Restructuring: } \\
\hline pre 2007/07/17 & 0.27 & 0.19 & 0.14 & 0.11 & 0.09 & 0.07 & 0.06 & 0.05 & 0.04 \\
\hline 2007/07/17-2008/03/16 & 0.26 & 0.16 & 0.13 & 0.11 & 0.09 & 0.08 & 0.07 & 0.06 & 0.05 \\
\hline 2008/03/16-2008/09/15 & 0.28 & 0.15 & 0.12 & 0.10 & 0.08 & 0.07 & 0.07 & 0.06 & 0.05 \\
\hline 2008/09/15 - & 0.29 & 0.17 & 0.12 & 0.10 & 0.09 & 0.08 & 0.06 & 0.05 & 0.05 \\
\hline
\end{tabular}

To study the impact of the restructuring mechanism on the FSD distributions, Table 4 displays the FSD distributions of the European CDS data according to their restructuring mechanism. Aggregated FSD distributions are displayed for 4 different time periods that are identical to the vertical lines in Figure 2: the period before the financial crisis (pre-2007/07/17), the time between 2007/07/17 and the fire-sale of Bear Stearns (2008/03/16); the time between 2008/03/16 and the Lehman collapse (2008/09/15) and the post-Lehman period. We can infer from Tables 4 and 5 that we observe roughly the same digit distributions for all four restructuring mechanisms, which illustrates that the influence of restructuring mechanisms is limited on the FSD distributions, but the regional differences are obvious again.

\section{Conclusion and Discussion}

In this article, we study Benford-like distributions for the CDS market during the last financial crisis. First, we illustrated the usefulness of such distributions to study the data quality of the CDS market. For the US and European markets, the data are consistent with the patterns of the corresponding Benford-like distributions. In studying the behavior of FSD distributions during the financial crisis 2007, we find huge fluctuations in the FSD for the European market, whereas the US market remains more or less constant. In the search for possible reasons, we inspect the FSD distributions for subsamples of data according to their creditworthiness or restructuring clause and show that these segmentations do not deliver sufficient explanation for the observed FSD patterns 
One possible reason for the observed differences could be that the European market was confronted with herding behavior during the crisis (Devenow \& Welch, 1996) whereby market participants make their decisions by imitating the actions (prices) of others due to the increasing market uncertainty. The absence of herding in the US market may be traced back to Chapter 11 of the Bankruptcy Code for US companies. In case of bankruptcy, Chapter 11 is lenient on debtors. Given an agreement with creditors, chapter 11 abdicates from liquidation and allows a debtor to continue and restructure its business. As a consequence, reorganization rates of bankruptcy are much higher in the US than in Europe, where such a restructuring clause is missing; however, in recent years several European countries have tried to change their bankruptcy norms in favor of the survival of companies. Where CDS are used as (hedging) instruments to protect against default risk in case of chapter 11 defaults, the protection seller is more flexible to recover claims from the reference entity. Our results nourish the notion that especially in stormy economic periods, the chapter 11 clause may affect the pricing mechanism. The absence of chapter 11 for European companies might imply that CDS spreads for European companies are more vulnerable to pricing malfunctions like herding. Following (Trichet, 2007) and (Park \& Sabourian, 2011), in times of economic uncertainty, herding is an immanent problem in financial markets. Building on the work of Jorion and Zhang (2007), who discuss contagion effects and the US bankruptcy clauses, this article sets the scene for further research, e.g., an investigation of herding in financial markets using FSD distributions.

\section{References}

Benford, F. (1938). The law of anomalous numbers. Proceedings of the American Philosophical Society, 78(4), 551-572.

Clippe, P., \& Ausloos, M. (2012). Benford's law and Theil transform of financial data. Physica A: Statistical Mechanics and its Applications, 391(24), 6556-6567.

Corazza, M., Ellero, A., \& Zorzi, A. (2010). Checking financial markets via Benford's law: the S\&P 500 case. In M. Corazza \& C. Pizzi (Eds.), Mathematical and statistical methods for actuarial sciences and finance ( pp. 93 - 102). Milano: Springer.
DeCeuster, M. J. K., Dhaene, G., \& Schatteman, T. (1998). On the hypothesis of psychological barriers in stock markets and Benford's Law. Journal of Empirical Finance, 5(3) , 263-279.

Dechert LLP. (2008, December 30). Derivatives Developments: Tackling the $\$ 50$ Trillion Credit Default Swap Market and Beyond. Retrieved from http://www.dechert.com/Derivatives_developments_tackling_the_50_trillion_credit_default_ swap_market_and_beyond_12-01-2008/

Depken, C. A. (2008). Benford, Zipf and the blogosphere. Applied Economics Letters, 15(9) , 689692. Devenow, A., \& Welch, I. (1996). Rational herding in financial economics. European Economic Review, 40(3-5), 603-615.

Forte, S., \& Peña, J. I. (2009). Credit spreads: An empirical analysis on the informational content of stocks, bonds, and CDS. Journal of Banking and Finance, 33(11), 2013-2025.

George, J., \& Laura, S. (2009). Detecting problems in survey data using Benford's Law. The Journal of Human Resources, 44(1), 1-24.

Giles, D. E. (2007). Benford's Law and naturally occurring prices in certain ebay auctions. Applied Economics Letters, 14(3), 157-161.

Greenspan, A. (2005, May 5). Remarks by Chairman Alan Greenspan. Retrieved from http://www.federalreserve.gov/Boarddocs/Speeches/2005/20050505

Grendar, M., Judge, G. G., \& Schechter, L. (2007). An empirical non-parametric likelihood family of data-based Benford-like distributions. Physica A: Statistical Mechanics and its Applications, 380(1 July 2007), 429-438.

Günnel, S., \& Todter, K. H. (2009). Does Benford's Law hold in economic research and forecasting. Empirica, 36(3), 273-292.

Hill, T. P. (1995). A statistical derivation of the significant-digit law. Statistical Science, 10(4), 354-363.

Jorion, P., \& Zhang, G. (2007). Good and bad credit contagion: Evidence from credit default swaps. Journal of Financial Economics, 84(3), 860-883.

Kuiper, N. H. (1960). Tests concerning random points on a circle. Proceedings of the Koninklijke Nederlandse Akademie van Wetenschappen - Series A, 63, 38-47.

Ley, E. (1996). On the peculiar distribution of the U.S. stock indexes' digits. The American Statistician, 50(4), 311-313. 
Longstaff, F. A., Mithal, S., \& Neis, E. (2005). Corporate yield spreads: Default risk or liquidity? New evidence from the credit default swap market. The Journal of Finance, 60(5), 2213-2253.

Park, A., \& Sabourian, H. (2011). Herding and contrarian behavior in financial markets. Econometrica, 79 (4), 973-1026.

Realdon, M. (2008). Credit default swap rates and stock prices. Applied Financial Economics Letters, 4(4), 241-248.

Scott, P., \& Fasli, M. (2001). Benford's Law: An empirical investigation and a novel explanation. Unpublished Manuscript.

Trichet, J. C. (2007, April 18). Some reflections on the development of credit derivatives. Retrieved from http://www.ecb.int/press/key/ date/2007/html/sp070418.en.html

Tukey, J. W. (1972). Some graphic and semigraphic displays. In T. A. Bancroft (Ed.), Statistical Papers in Honor of George W. Snedecor (pp. 293-316). Arnes, IA: Iowa State University Press.

Varian, H. R., Morgan, J. A., Deaton, A. S., Cramer, E. M., \& Bibby, J. (1972). Letters to the editor. The American Statistician, 26(3), 62-65.

\section{Acknowledgments}

The first author's research is supported by the Oesterreichische Nationalbank under the Jubilaumsfond grant 14663. 
